Eurostudia

\title{
Gouverner le religieux dans l'espace européen et nord-américain : entre acteurs politiques, judiciaires et religieux
}

\section{Claude Proeschel et Sylvie Toscer-Angot}

Volume 13, numéro 1-2, 2018-2019

Cultures en contact, entre régulations et représentations

URI : https://id.erudit.org/iderudit/1064486ar

DOI : https://doi.org/10.7202/1064486ar

Aller au sommaire du numéro

Éditeur(s)

Le Centre canadien d'études allemandes et européennes

ISSN

1718-8946 (numérique)

Découvrir la revue

Citer ce document

Proeschel, C. \& Toscer-Angot, S. (2018). Gouverner le religieux dans l'espace européen et nord-américain : entre acteurs politiques, judiciaires et religieux. Eurostudia, 13(1-2), 19-26. https://doi.org/10.7202/1064486ar

Tous droits réservés ( Le Centre canadien d'études allemandes et européennes, 2019
Ce document est protégé par la loi sur le droit d'auteur. L'utilisation des services d'Érudit (y compris la reproduction) est assujettie à sa politique d'utilisation que vous pouvez consulter en ligne.

https://apropos.erudit.org/fr/usagers/politique-dutilisation/ 
Gouverner le religieux dans l'espace européen et nord-américain: entre acteurs politiques, judiciaires et religieux

\section{Claude Proeschel}

GSRL (EPHE-CNRS), PSL Research University

Sylvie Toscer-Angot

Université Paris-Est Créteil Val-de-Marne;

GSRL (EPHE-CNRS), PSL Research University

\section{Le religieux, un domaine de l'action publique}

La relation entre action publique et problèmes publics est beaucoup plus complexe que ne le suggère l'idée commune selon laquelle les politiques serviraient à résoudre les problèmes (Muller 2000 : 194).

L'inscription de la question religieuse à l'agenda public, qui s'est généralisée dans les années 1990 dans l'ensemble des démocraties occidentales, et qui, « développement impensable il y a quelques années », s'y "maintient " (Côté et Gunn 2006) semble bien s'inscrire dans la logique de l'approche cognitive. Elle ne constitue en effet pas seulement ou pas prioritairement une réponse à un ensemble de problèmes objectifs nécessitant la mise en place d'une action politique. Elle résulte aussi, voire avant tout, d'une évolution de la perceptions des situations par les pouvoirs publics. 
On peut alors la considérer comme la conjonction de deux phénomènes. Tout d'abord, si la plupart des sociologues s'accordent à inscrire les sociétés européennes dans des logiques longues de sécularisation, qui s'expriment à la fois par un «déclin de l'hégémonie religieuse » (Monod 2007) et une "sortie de la religion » (Gauchet 1985), celles-ci sont cependant l'objet d'un retour contemporain de la présence du religieux dans l'espace public (Casanova 1994). Ses expressions, protéiformes, recouvrent par exemple, les débats sur l'institutionnalisation de l'islam (Frégosi 2008; Cesari 2009; Thomas 2012), l'intervention de courants identitaires catholiques (Portier 2012) dans les débats sur des projets de lois sociétaux (Dobbelaere et Pérez-Agote 2015), mais aussi l'accroissement sensible des controverses relatives à des questions aussi diverses que la construction de lieux de culte, la présence de symboles religieux dans les lieux publics, les normes vestimentaires, l'abattage rituel, ou encore la pratique religieuse dans le cadre professionnel.

Cette situation, qui a conduit à l'élaboration, dans le monde académique, d'une interrogation sur la notion de sécularisation comme processus linéaire et continu (Berger 1999), ne peut, à elle seule, expliquer la perpétuation des politiques publiques du religieux. « Les variations de l'environnement » ne devenant en effet «des faits significatifs que dans la mesure où elles peuvent être nommées et interprétées » (Jobert 1992 : 220), elle a également trouvé source dans l'évolution de la façon dont les pouvoirs publics se représentent les enjeux des questions religieuses. Faire une politique publique, en effet, ce n'est pas "'résoudre' un problème, mais construire une nouvelle représentation de ce problème qui met en place les conditions sociopolitiques de son traitement par la société » (Muller et Surel 1998: 31). Ainsi la remise en cause de la contestation au sein de la communauté scientifique de la thèse de la sécularisation, dans les années 1990, et du lien automatique modernité-sécularisation a marqué l'action publique et contribué à l'évolution de son référentiel. La religion n'est plus désormais 
considérée comme une simple survivance, mais comme un domaine de gestion des gouvernements démocratiques, dont les enjeux en évolution, en termes d'intégration, de vivre-ensemble ou de neutralité, se doivent de faire l'objet d'une régulation (Barras, Dermange et Nicolet 2017).

\section{Qui gouverne le religieux?}

Cette régulation du religieux est marquée, de manière plus récente, par une évolution dans ses niveaux d'exercice, dorénavant pluriels (local, national, international ou supranational), et dans la diversification des catégories d'acteurs y contribuant : politiques, judiciaires, acteurs de la société civile, qui interagissent et interfèrent mutuellement. Ces nouveaux modes de décision publique et de coordination de l'action publique, pour lesquels le cadre classique de «gouvernement», relié au monopole de l'Etat, ne semble plus adapté, renvoie alors davantage le chercheur vers la notion de gouvernance, entendue comme une « interaction d'une pluralité d'acteurs gouvernants qui ne sont pas tous étatiques ni même publics » (Leca 1996 : 339).

La notion de gouvernance s'est imposée dans l'analyse des politiques publiques dans les années 1980, même si elle a un ancrage plus ancien dans le domaine du management des entreprises, à travers la notion de «corporate governance » (Coase 1937). On la définit généralement comme un "processus de coordination d'acteurs, de groupes sociaux, d'institutions, pour atteindre des buts propres discutés et définis collectivement dans des environnements fragmentés et incertains » (le Galès 1999) ou encore comme « les nouvelles formes interactives de gouvernement dans lesquelles les acteurs privés, les différentes organisations publiques, les groupes ou communautés de citoyens, ou d'autres types d'acteurs, prennent part à la formulation de la politique » (Marcou 1997). 
Elle se veut donc un outil d'appréhension et d'explication de l'évolution des modes décisionnels, dans leur multiplication et leur complexification, vers un polycentrisme institutionnel (Boussaguet 2010), mais aussi sur un mode de fonctionnement plus horizontal et moins coercitif des processus d'autorité au sein de ces constellations d'acteurs (Gräsel et al. 2011).

La gouvernance, on le voit, ne constitue pas un concept, ni une théorie, mais bien une notion, qui veut permettre avant tout de poser de manière adéquate le questionnement de situations nouvelles. C'est à celui-ci que s'attachent les différentes contributions composant ce numéro, qui explore différentes facettes de la gouvernance du religieux. Elles soulignent en particulier trois phénomènes : la complexité des liens et des interactions entre les acteurs qui sont partie prenante de la régulation; le poids, certes, des héritages à la fois religieux et politiques dans l'évolution des modes de gouvernance, mais aussi l'existence de possibles décalages entre les discours, ancrés dans des récits marqués par une forte « dépendance au sentier », et la réalité des pratiques.

Louis Léon Christians, dans sa contribution, s'intéresse aux effets de la dualité, voire de la concurrence des ordres normatifs européens concernant les questions religieuses, sur la gouvernance multi-niveaux des questions religieuses, en particulier du pluralisme, et leurs évolutions récentes.

Elise Massicard souligne, quant à elle, la forte prégnance du contrôle étatique de la gestion du religieux en Turquie. Dans son étude des évolutions consécutives à l'arrivée d'un parti islamique au pouvoir, elle montre comment celui-ci, voulant limiter l'influence à la fois de la CEDH et d'institutions encore dominées par la logique kémaliste, use de «la religion comme politique publique ». 
Jean-François Husson montre comment le financement de cultes s'insère dans une volonté de co-régulation initiée par l'Etat belge, qui, en assurant aux religions les conditions financières de leur déploiement social, les met à même d'assurer le rôle de cohésion sociale ou d'ordre public qu'il leur prête. Pour autant, l'analyse des modalités concrètes de cette gouvernance instituée par les autorités politiques dévoile des pratiques infléchies par le niveau local.

Nicolas Kazarian interroge ici les effets des récents phénomènes migratoires sur les débats autour de la gestion du pluralisme en Grèce, et montre comment celui-ci est traversé par des éléments souvent contradictoires : le sens des normes européennes et sa réception politique, et une tradition fortement marquée par le contexte historique et religieux orthodoxe, souvent mobilisée par des courants identitaires contre les migrants, à rebours de l'investissement concret de l'Eglise orthodoxe dans la gestion de la crise migratoire.

Goran Sekulovski aborde la question de la gouvernance du religieux en Macédoine via un angle peu exploré, celui de l'occupation de l'espace par la construction de bâtiments cultuels, qui, souvent, ne correspondent pas à des besoins objectifs, financés à la fois par les autorités politiques ou les acteurs de la société civile, en particulier les acteurs religieux.

La question des signes religieux, qui renvoie à la fois aux problématiques de neutralité, de conceptions du pluralisme, et donc à des dimensions à la fois politiques et juridiques, est un indicateur éclairant des modes de régulation du religieux. A partir du cas du port du foulard islamique par les enseignantes, Sylvie Toscer-Angot montre comment, en ce domaine, s'entremêlent le juridique et le politique, parfois au profit du premier, et comment l'existence des niveaux fédéral et fédéré permet des gouvernances modulées, la religion étant de la compétence des Länder. 
Blandine Chélini-Pont, dans son étude des politiques publiques du religieux aux Etats Unis, souligne comment celles-ci résultent d'équilibres souvent redéfinis entre les différents pouvoirs, exécutif et législatif, au sein du pouvoir législatif et dans le jeu des réactions entre décisions politiques et judiciaires, du fait de la place forte faite au juge dans la régulation de la société américaine.

\section{Bibliographie}

Barras, A., F. Dermange et S. Nicolet (dir.). (2016). Réguler le religieux dans les sociétés libérales. Genève, Éditions Labor et Fides.

Berger, P. (dir.). (1999). The Desecularization of the World, Resurgent Religion and World Politics. Grand Rapids (MI), Eerdmans.

Boussaguet, L., S. Jacquot et P. Ravinet. (2010). Dictionnaire des politiques publiques. Paris, Presses de Sciences Po.

Bréchon, P. et F. Gonthier (dir). (2014). Les valeurs des Européens. Evolutions et clivages. Paris, Armand Colin.

Casanova, J. (1994). Public Religions in the Modern World. Chicago, University of Chicago Press.

Cesari, J. (2009). Muslims in the West after 9/11. Religion, Politics and Law. Londres, Routledge.

Coase, R. (1937). « The Nature of the Firm ». Economica, vol. 4, n¹6, p. 386-405.

Côté, P. et J. Gunn (dir.). (2006). La nouvelle question religieuse. Régulation ou ingérence de l'État? Dieux, Hommes et Religions. Bruxelles, Peter Lang. 
Davie, G. (2002). Europe: the Exceptional Case. Parameters of Faith in the Modern World. Londres, Darton, Logman and Todd.

Dobbelaere, K. et A. Pérez-Agote (dir). (2015). The Intimate. Polity and the Catholic Church: Laws about Life, Death and the Family in So-called Catholic Countries. Leuven, Leuven University Press.

Frégosi, F. (2008). Penser l'Islam dans la laïcité: les musulmans de France et la République. Paris, Fayard.

Gauchet, M. (1985). Le désenchantement du monde. Une histoire politique de la religion. Paris, Gallimard.

Gräsel, C., I. Bormann, K. Schütte, K. Trempler, R. Fischbach et R. Assenburg. (2011). " Outlook on research in education for sustainable development». Policy Futures in Education, vol 13, n², p. 115-127.

Jobert, B. (1992).«Représentations sociales, controverses et débats dans la conduite des politiques publiques ». Revue française de science politique, vol 42, $\mathrm{n}^{\circ}$, p. 219-34.

Leca, J. (1996). «La "gouvernance" de la France sous la Cinquième République ». Dans F. D'Arcy et L. Rouban (dir), De la Cinquième République à l'Europe. Paris, Presses de Sciences Po, p. 329-365.

Le Galès, P. (1999). «Régulation, gouvernance et territoires ». Dans J. Commaille et B. Jobert (dir.), Les Métamorphoses de la régulation politique. Paris, Librairie générale de droit et de jurisprudence (LGDJ), p. 203-239.

Marcou, G., F. Rangeon et J.-L. Thiebault. (1997). « Les relations contractuelles entre collectivités publiques». Dans F. Godard (dir.), Le gouvernement des villes. Territoire et pouvoir. Paris, Ed. Descartes \& Cie, p. 137-211.

Monod, J.-C. (2007). Sécularisation et laïcité. Paris, PUF.

Muller, P. et Y. Surel. (1998). L'analyse de politiques publiques. Paris, Montchrestien.

Muller, P. (2000). "L'analyse cognitive des politiques publiques: vers une sociologie politique de l'action publique ». Revue française de science politique, vol. 50, n², p. 189-208. 
26 Gouverner le religieux

Portier, P. (2012). «Pluralité et unité dans le catholicisme français ». Dans C. Béraud, F. Gugelot et I. Saint-Martin (dir.), Catholicisme en tension. Paris, Editions de l'EHESS, p. 19-35. 